\title{
Morphological Wetting Transitions at Ring-Shaped Surface Domains
}

\author{
Claudia Schäfle, ${ }^{\dagger}$ Martin Brinkmann, ${ }^{*}$ Clemens Bechinger ${ }^{\S}$ Paul Leiderer," and \\ Reinhard Lipowsky*, \\ ${ }^{\dagger}$ Fachhochschule Rosenheim, Fakultät für Angewandte Natur- und Geisteswissenschaften, Hochschulstrasse I, \\ 83024 Rosenheim, Germany, "Max Planck Institute for Dynamics and Self-Organization, Bunsenstr. 10, \\ 37073 Göttingen, Germany, ${ }^{8}$ Fachbereich Physik, Universität Stuttgart, Pfaffenwaldring 57, 70550 Stuttgart, \\ Germany, "Fachbereich Physik, Universtät Konstanz, Universitätsstrasse 10, 78457 Konstanz, Germany, and \\ ${ }^{\perp}$ Max Planck Institute of Colloids and Interfaces, 14424 Potsdam, Germany
}

\begin{abstract}
The wetting behavior of ring-shaped (or annular) surface domains is studied both experimentally and theoretically. The ring-shaped domains are lyophilic and embedded in a lyophobic substrate. Liquid droplets deposited on these domains can attain a variety of morphologies depending on the liquid volume and on the dimensions of the ringlike surface domains. In the experiments, the liquid volume is changed in a controlled manner by varying the temperature of the sample. Such a volume change leads to a characteristic sequence of droplet shapes and to morphological wetting transitions between these shapes. The experimental observations are in good agreement with analytical and numerical calculations based on the minimization of the interfacial free energy. Small droplets form ringlike liquid channels (or filaments) that are confined to the ring-shaped domains and do not spread onto the lyophobic disks enclosed by these rings. As one increases the volume of the droplets, one finds two different morphologies depending on the width of the ring-shaped domains. For narrow rings, the droplets form nonaxisymmetric liquid channels with a pronounced bulge. For broad rings, the droplets form axisymmetric caps that cover both the lyophilic rings and the lyophobic disks.
\end{abstract}

\section{Introduction}

During the past decade, an increasing number of wetting experiments has been performed for substrate surfaces which exhibit patterns of lyophilic and lyophobic surface domains. Such surfaces can be easily obtained by microcontact printing, ${ }^{1}$ and by other methods such as vapor deposition of inorganic substances through masks used in electron microscopy ${ }^{2}$ or self-assembled colloids, ${ }^{3,4}$ which lead to surface domains with a linear size from many micrometers to about a hundred nanometers. Even smaller domain sizes are accessible using controlled oxidation of $\mathrm{Si}$ substrates in the presence of an atomic force microscopy (AFM) tip by which one can create patterns of lyophilic spots with a spacing of a few nanometers. ${ }^{5}$ During the last couple of years, these patterning methods have been further developed and new patterning

*To whom correspondence should be addressed. E-mail: lipowsky(a mpikg.mpg.de.

(1) Xia, Y.; Whitesides, G. M. Soft lithography. Angew. Chem. 1988, 37, 550575 .

(2) Gau, H.; Herminghaus, S.; Lenz, P.; Lipowsky, R. Liquid morphologies on structured surfaces: From microchannels to microchips. Science 1999, 283, 46-49.

(3) Burmeister, F.; Schäfle, C.; Matthes, T.: Böhmisch, M.; Boneberg. J.; Leiderer. P. Colloid monolayers as versatile lithographic masks. Lamgmuir 1997, 13, 2983P. Col
2987.

(4) Drodofsky, U.; Schulze, T.; Stuhler, J.; Drewsen, M.; Brezger, B.; Pfau, T.; Mlynek, J. Hexagonal nanostructures generated by light masks for neutral atoms. Appl. Phiss. B: Laser Opt. 1997, 65, 755-759.

(5) Gareia, R.: Calleja, M.: Perez-Murano, F. Local oxidation of silicon surfaces by dynamic force microscopy: Nanofabrication and water bridge formation. Appl. Phys. Lell 1998, 72,2295-2297.

(6) Zhang, X.; Jin, M.; Liu, Z.; Tryk, D. A.; Nishimoto, S.; Murakami, T.; Fujishima, A. Superhydrophobic $\mathrm{TiO}_{2}$ surfaces: Preparation, photocatalytic wettability conversion, and superhydrophobic-superhydrophilic patterning. J. Phys Chem C 2007, III, 14521-14529

(7) Zeira, A.; Chowdhury, D.: Maoz, R.; Sagiv, J. Contact electrochemical replication of hydrophilic-hydrophobic monolayer patterns. ACS Nano 2008, 2. $2554-2568$.

(8) Park, S. K.; Kim,Y.-H.; Han, J.-I. High-resolution patterned nanoparticulate $\mathrm{Ag}$ electrodes toward all printed organic thin film transistors. Org. Electron. 2009, 10, 1102-1108. protocols have been introduced: ${ }^{6-10}$ recent reviews of these methods include refs 11 and 12 .

Wetting processes at such chemically patterned surfaces raise fundamental questions about the wetting morphologies and are essential for many applications in the field of microfluidics. One particularly simple wetting geometry consists of striped lyophilic domains within a lyophobic substrate, a geometry that can lead to long liquid channels, which spread along the stripes, or to dropletlike structures, which are localized over short segments of the stripes. If the stripes are completely covered by the wetting liquid, the two ends of the channel have a fixed position which is determined by the two ends of the underlying stripe. ${ }^{2}$ If the stripes are only partially covered by the liquid, the two ends can now move along the stripe. ${ }^{13}$ Both situations lead to morphological wetting transitions. Analogous wetting phenomena are also observed for liquid droplets at topographically structured substrates as studied in refs 14 and 15 .

Wetting morphologies in the micrometer range have the same shape as macroscopic droplets in the absence of gravity. If a certain amount of liquid is placed on a chemically homogeneous and planar

(9) Chen, C.-T.; Tseng, Z.-F.; Chiu, C.-L.; Hsu, C.-Y.; Chuang, C.-T. Selfaligned hemispherical formation of microlenses from colloidal dioplets on heterogeneous surfaces. J. Micromech. Microeng. 2009, 19, 025002.

(10) Bliznyuk, O.: Vereshchagina, E.; Kooij, E. S.: Poelsema, B. Scaling of anisotropic droplet shapes on chemicaly stripe-patterned suffaces. Phys. Rev. $E$ 2009, 79, 041601 .

(11) Woodson. M.; Liu. J. Functional nanostructures from surface chemistry patterning. Phis. Chem. Chem. Phys. 2007, 9, 207-225.

(12) Nie, Z,; Kumacheva, E. Patterning surfaces with functional polymers. Nal. Matter. 2008, 7, 277-290

(13) Brinkmann, M.; Lipowsky, R. Wetting morphologies on substrates with striped surface domains. J. Appl. Phss. 2002, 92, 4296-4306.

(14) Seemann, R.: Brinkmann, M.: Kramer, E. J.; Lange, F. F.: Lipowsky, R. Wetting morphologies at microstructured surfaces. Proc. Natl. Acad. Sci. U.S.A. $2005,102,1848-185 ?$

(15) Semprebon, C.: Mistura, G.: Orlandini, E.; Bissaco. G.; Segato, A.; Yeomans, J. M. Anisotropy of water droplets on single rectangular posts. Langmuir 2009, 25, $5619-5625$ 
substrate, it attains the unique equilibrium shape of a spherical cap which is the only mechanically stable state. ${ }^{16}$ In general, the shape of the vapor-liquid or fluid-fluid interface, which is in mechanical equilibrium, must correspond to a surface of constant mean curvature $M$. Furthermore, this interface forms a certain contact angle $\theta$ with the substrate surface along the contact line, that is, along the three-phase line where the vapor phase $(\alpha)$, the liquid phase $(\beta)$, and the substrate $(\sigma)$ meet.

The condition of constant mean curvature follows from the well-known Laplace equation, $2 \Sigma_{\alpha \beta} M=P_{\beta}-P_{\alpha}$, relating the mean curvature $M$ of the $(\alpha \beta)$ interface to its surface tension $\Sigma_{\alpha \beta}$ and the pressures $P_{\alpha \alpha}$ and $P_{\beta}$ within the adjacent bulk phases $(\alpha)$ and $(\beta)$, which may be a liquid and a vapor phase or two liquid phases. In equilibrium, the surface tension $\Sigma_{\alpha \beta}$ is the same for all patches of the interface. Likewise, the pressure difference $P_{\beta}-P_{\alpha}$ does not depend on the position of the $(\alpha \beta)$ interface provided one can ignore the effects of gravity as assumed here. The latter assumption implies that the size of the droplet is significantly smaller than the capillary length $\xi_{\text {cap }}$ which is about $2 \mathrm{~mm}$ for the air/water interface at room temperature.

The contact angle $\theta$ at the contact or three phase line is determined by the balance of forces arising from the interfacial tensions at the contact line. Since the substrate $(\sigma)$ is taken to be rigid, the tangential components of these forces have to cancel out in mechanical equilibrium. For a chemically homogeneous substrate, this force balance leads to a constant contact angle $\theta$ which satisfies the classical Young-Dupré equation $\Sigma_{\alpha \beta} \cos \theta=\Sigma_{\alpha, \sigma}-\Sigma_{\beta \sigma}$

In principle, one can imagine other liquid morphologies on chemically homogeneous and planar substrates which still have

a constant contact angle. One example is provided by a liquid channel, that is, a cylindrical segment the axis of which lies parallel to the surface and which satisfies periodic boundary conditions in this direction. Such a channel becomes unstable if its aspect ratio exceeds a certain threshold value as shown in refs 17 and 18 for small and arbitrary contact angles, respectively.

Chemically Heterogeneous Substrates. In contrast to a homogeneous substrate surface, a patterned surface, which contains lyophilic and lyophobic surface domains, can exhibit a large variety of different equilibrium shapes. Examples are provided by (i) one large droplet coexisting with many small ones on an array of circular lyophilic domains, ${ }^{19}$ (ii) liquid channels on striped lyophilic domains ${ }^{2,13}$ or films with stable holes induced by circular lyophobic domains. ${ }^{20}$ In all of these cases, the contact line is partially or fully pinned to the boundary between the lyophilic domains $(\gamma)$ and the lyophobic domains $(\delta)$. For a pinned segment of the contact line, the local contact angle $\theta$ satisfies the inequalities $\theta_{\gamma} \leq \theta \leq \theta_{\delta}$ where $\theta_{\gamma}$ and $\theta_{\delta}$ are the local contact angles which the $\alpha \beta$ interface attains in equilibrium on the $(\gamma)$ and $(\delta)$ domains, respectively. ${ }^{19}$ 781

(16) Finn, R. Capillary surface interfaces. Not. Am. Math. Soc. 1999, 46, 770-

(17) Sekimoto, K.; Oguma, R.: Kawazaki, K. Morphological stability analysis of partial wetting. Ann. Phys. 1987, 176, 359-392

(18) Lenz. P.: Lipowsky, R. Stability of droplets on homogeneous and struc(18) Lenz, P.; Lipowsky, R. Stability of droplet
tured surfaces. Euro. Phys. J. E 2000, I, 249-262.

(19) Lenz, P.; Lipowsky, R. Morphological transitions of wetting layers on structured surfaces. Phys. Rev. Lett. 1998, 80, 1920-1923.

(20) Lenz, P.; Bechinger, C.; Schäfle, C.; Leiderer, P.; Lipowsky, R. Perforated wetting layers from periodic patterns of lyophobic surface domains. Langmuir 2001. $17,7814-7822$.

(21) Darhuber, A. A.; Trojan, S. M.; Miller, S. M.; Wagner, S. Morphology of liquid microstructures on chemically patterned surfaces. J. Appl. Phys. 2000, 87, $7768-7775$.
The behavior of liquid placed on striped lyophilic surface domains has been studied in some detail, both experimentally ${ }^{2,21}$ and theoretically. ${ }^{2,13.18}$ In the case of large lyophobic contact angles, the liquid will form channels on the lyophilic stripe if the lyophilic contact angle is sufficiently small. Depending on the length of the lyophilic stripe and the volume of the droplet, the liquid might cover the stripe completely or only partially. (This should be distinguished from the usual terminology of complete and partial wetting which corresponds to $\theta_{\nu}=0$ and $\theta_{\nu}>0$, respectively. For $\theta_{\gamma}=0$, the stripe must be covered completely by the wetting liquid. However, for $\theta_{\gamma}>0$, the stripe is partially covered for small liquid volumes but completely covered for large liquid volumes.) If the liquid covers the stripe completely, no segment of the contact line is located within the lyophilic $(\gamma)$ domain, and the local contact angle $\theta$ differs from $\theta_{\gamma}$ for all contact line segments. If the liquid covers the stripe partially, the two ends of the channel are bounded by two contact line segments with $\theta=\theta_{\gamma}$.

The channel may undergo a morphological wetting transition both for complete ${ }^{2}$ and for partial ${ }^{13}$ coverage of the striped surface domain. The general bifurcation diagram for the wetting morphologies has been determined in ref 13. In addition to the line of morphological wetting transitions, at which extended channel states coexist with localized droplet states, this diagram contains two instability lines at which the channel and droplet states become unstable, respectively. Long channels become unstable as soon as the local contact angle $\theta$ along the pinned segments of the contact line exceeds the critical value $\theta=\theta^{*}=90^{\circ}$. ${ }^{2.13,18}$

In the present Article, we study the wetting of ring-shaped or annular surface domains which extends previous studies as reported in refs 22 and 23 to the micrometer regime. Our experimental system allows us to vary the volume of the adsorbed liquid continuously and, in this way, induce and study morphological transitions of the wetting droplets. After a certain amount of liquid has been deposited on the annular surface domain, a ringlike channel with rotational symmetry is formed. As the volume is further increased, this channel undergoes an abrupt transition toward a bulgelike shape with broken rotational symmetry provided the width of the annular domain is sufficiently small. As one adds more liquid, the bulge continues to grow until it undergoes a second transition toward a spherical cap which covers both the lyophilic annulus and the inner lyophobic domain completely. For broader rings, one has a single transition from the axisymmetric channel to the spherical cap without an intermediate bulge state. The experimental system is sufficiently robust so that the shape of the liquid can be determined by AFM. Our experimental observations are compared with detailed analytical and numerical calculations. In addition, we present the complete morphological bifurcation diagram for the lyophilic annulus. Our results are consistent with and provide a classification scheme for the droplet morphologies as found for a lattice model using density functional theory and Monte Carlo simulations. ${ }^{24}$

\section{Experimental Procedures}

Chemically patterned substrates were fabricated by microcontact printing $(\mu \mathrm{CP})^{25}$ of alkanethiols on gold coated glass sheets.

(22) Lenz, P.. Fenzl, W.; Lipowsky. R. Wetting of ring-shaped surface domains. Europhys. Lett. 2001, 53.618-624

(23) Jokinen, V.; Sainiemi, L.; Franssila, S. Complex droplets on chemically modified silicon nanograss. Adv. Mater. 2008, 20,3453-3456.

(24) Porcheron, F. Monson, P. A. Schoen. M. Wetting of rings on a nanopatterned surface: A lattice model study. Phys. Rev. E 2006, 73, 041603 
Since this technique has become well established in recent years, ${ }^{\prime}$ it will be sufficient to give a brief summary of our experimental preparation procedures. We printed a $1 \mathrm{mM}$ ethanolic solution of nonpolar octadecanethiol $\mathrm{HS}\left(\mathrm{CH}_{2}\right)_{17}-\mathrm{CH}_{3}$ with a patterned stamp of elastomeric material (poly(dimethylsiloxane), PDMS) onto the substrate and subsequently dipped the substrate in a $1 \mathrm{mM}$ ethanolic solution of polar 11-mercaptoundecanol $\mathrm{HS}\left(\mathrm{CH}_{2}\right)_{11}{ }^{-}$ $\mathrm{OH}$. In this way, we constructed substrate surfaces consisting of annular lyophilic surface domains within a lyophobic matrix. The resulting surface domain structures were characterized by monitoring the phase signal during imaging of the surface using AFM in tapping mode.

Two different annular geometries were used: (i) narrow rings with outer radius $r=(3.75 \pm 0.05) \mu \mathrm{m}$ and inner radius $r=$ (2.50 \pm 0.05$) \mu \mathrm{m}$, and (ii) broad rings with $r_{>}=(7.65 \pm 0.05) \mu \mathrm{m}$ and $r_{<}=(2.25 \pm 0.05) \mu \mathrm{m}$. The aspect ratio

$$
a \equiv r_{<} / r_{>}
$$

of the inner to the outer radius is $a=0.667 \pm 0.016$ for the narrow rings and $a=0.294 \pm 0.007$ for the broad rings. The corresponding width

$$
L \equiv r_{>}-r_{<}
$$

of the narrow and broad rings is $L=(1.25 \pm 0.08) \mu \mathrm{m}$ and $L=$ $(5.40 \pm 0.08) \mu \mathrm{m}$, respectively.

Large droplets of sulfuric acid (96 wt \%) were placed on these chemically patterned surfaces. The liquid first covered the whole substrate and then started to dewet from the lyophobic $(\delta)$ domains while it stayed in contact with the lyophilic $(\gamma)$ domains. This led to a corrugation of the contact line and eventually to the formation of disconnected droplets until each droplet covered one annu$\operatorname{lar}(\gamma)$ domain together with the circular $(\delta)$ domain enclosed by the $(\gamma)$ annulus.

At this stage, the amount of deposited liquid is still too large to form ring-shaped channels which cover only the $(\gamma)$ domains. In order to reduce the amount of liquid, a flat PDMS surface was slowly moved toward the surface until it touched the liquid droplets. When the PDMS surface was removed again, it took away some liquid which adhered to it and, in this way, reduced the volume of the droplets. Direct inspection showed that this procedure leads to the formation of ring-shaped channels on several $(\gamma)$ domains.

The volume of the liquid channels prepared in this way was further changed by varying the temperature of the substrate When the substrate is cooled, the evaporation rate at the sulfuric acid/air interface decreases and the liquid volume increases by condensation of water from the ambient air. When the substrate is heated, on the other hand, the evaporation rate increases and the liquid volume decreases.

In this way, the dependence of the droplet morphology on its volume was studied by video microscopy. The temperature range in the presented example was between 12 and $28^{\circ} \mathrm{C}$, which corresponds to a change in concentration of the acid from 27 to $57 \mathrm{wt} \%$ This led to an estimated variation of the contact angle $\theta_{\delta}$ from approximately $102^{\circ}$ to $98^{\circ}$.

In addition, the different morphologies were scanned using AFM in tapping mode at ambient conditions $\left(T=23^{\circ} \mathrm{C}, 50 \mathrm{wt} \%\right.$ sulfuric acid). We used a commercial atomic force microscope (Nanoscope 3, Multimode, Digital instruments, Santa Barbara, CA) with Si cantilevers ( $v_{\mathrm{res}} \approx 325 \mathrm{kHz}$, NT-MDT, Moskow) in tapping mode at very low scan rates. For these conditions, the advancing contact angle was also measured optically which led to the estimates $\theta_{\delta}=(102 \pm 2)^{\circ}$ for the $\mathrm{CH}_{3}$-terminated thiols and $\theta_{\gamma}=(33 \pm 2)^{\circ}$ for the $\mathrm{OH}$-terminated thiols.

(25) Kumar. A.: Whitesides, G. M. Features of gold having micrometer to centimeter dimensions can be formed through a combination of stamping with an elastomeric stamp and an alkanethiol ink followed by chemical etching. Appl. Phys. Lett. 1993, 63, 2002-2004.

\section{Theoretical Description}

The size of the droplets studied in our experiments was in the micrometer regime. This implies a convenient separation of length scales which simplifies the theoretical modeling. ${ }^{26}$ On the one hand, the droplet size was always much smaller than the capillary length $\xi_{\text {cap }}$ of the interface between sulfuric acid and air which is $\xi_{\text {cap }} \simeq 2 \mathrm{~mm}$ for the temperatures and pressures considered here. In such a situation, one may safely ignore the effects of gravity. On the other hand, we may also ignore the effects of line tension $\Lambda$, which represents the excess free energy density of the contact line, since the corresponding crossover length $\Lambda / \Sigma_{\alpha \beta}$ is expected to be about $30-100 \mathrm{~nm} .{ }^{26}$ Likewise, the tension $\Sigma_{\alpha \beta}$ of the $\alpha \beta$ interface is so large that the thermally excited roughness scale $\sim\left(T / \Sigma_{\alpha \beta}\right)^{1 / 2}$ of this interface is of the order of a few angstroms which is completely negligible compared to the size of the droplets.

Using these simplifications, the shape of the $(\alpha \beta)$ interface corresponds to a surface of constant mean curvature as follows from the Laplace equation. Provided these shapes are axisymmetric, they can be parametrized by segments of Delauny's surfaces. In the situation considered here, axisymmetric channel states correspond to segments of nodoids. ${ }^{22}$ Likewise, the droplets, which cover both the lyophilic annulus and the interior lyophobic domain, correspond to spherical caps.

These constant mean curvature surfaces are bounded by contact lines which must fulfill certain boundary conditions. If the contact line lies within a $(\gamma)$ or within a $(\delta)$ domain, the local contact angle $\theta$ between the $(\alpha \beta)$ interface and substrate surface must have the values $\theta=\theta_{\gamma}$, and $\theta=\theta_{j}$, respectively. If the position of the contact line coincides with the position of a domain boundary between some $(\gamma)$ and $(\delta)$ domains, the contact angle $\theta$ is not fixed but can attain any value between $\theta_{\gamma}$ and $\theta_{\partial}{ }^{19}$

Constant mean curvature shapes which satisfy the Laplace equation represent states which correspond to extrema of the interfacial free energy. If one wants to determine the stable or metastable states, one must ensure that these free energy extrema are, in fact, free energy minima. This is achieved by a stability analysis of the constant mean curvature shapes. Such an analysis determines the free energy associated with small but arbitrary perturbations or distortions of the constant mean curvature shape. A convenient parametrization of these perturbations is as follows. ${ }^{27}$ Each point of the $(\alpha \beta)$ interface is displaced along the local surface normal with respect to the initial configuration. Using this parametrization, the second variation of the interfacial free energy becomes a quadratic form of the normal displacement field. The droplet will be stable or at least metastable if the associated quadratic form is positive for any variation of the droplet shape which leaves the volume unchanged up to linear order.

It can be shown that this problem is equivalent to the solution of an inhomogeneous eigenvalue problem involving an elliptic, second order, partial differential operator. ${ }^{27}$ In the calculus of variation, this equation is known as Jacobi's accessory differential equation. ${ }^{28}$ A subsidiary linear condition arises from the constraint of fixed droplet volume. The lowest eigenvalue to this eigenvalue problem determines the stability of the morphology: if this eigenvalue is positive, the configuration of the $(\alpha \beta)$ interface is stable and the shape corresponds to a (global or local) minimum of the interfacial free energy.

(26) Lipowsky, R.; Lenz. P.; Swain. P. Wetting and dewetting of structured and imprinted surfaces. Colloids Surf., A 2000, 16I, 3-22.

(27) Brinkman, M.; Kierfeld, J.; Lipowsky, R. A general stability criterion for droplets on structured substrates: The effect of contact line tension. J. Phys. A Math. Gen. 2004, 37, 11547-11573.

(28) Giaquinta, M.; Hildebrandt, S. Calculus of variations I and II; Springer: Berlin, 1996. 
1<smiles>c1ccccc1</smiles>

2

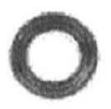

$\uparrow$

16

(a)<smiles>C1=C=C2C=CC=C(C=1)C2</smiles>

15

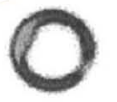

3

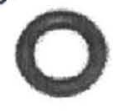

(b)

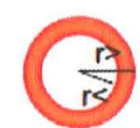

$V / L^{3}=0.5$

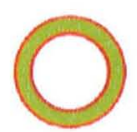

4
14
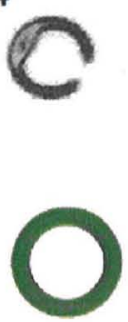

5.5

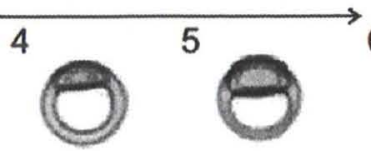

13
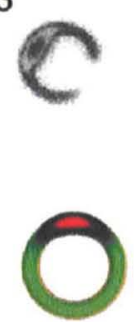

5.95
12
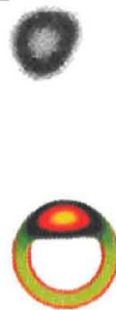

7
6

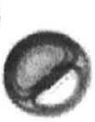

7

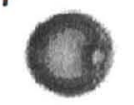

11
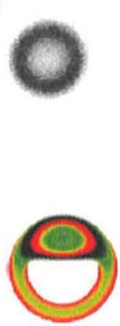

10
8

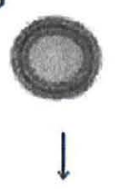

9

10

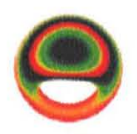

18

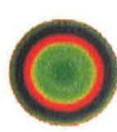

22

Figure 1. Wetting morphologies for a lyophilic ring-shaped (or annular) surface domain. (a) Droplet shapes of aqueous sulfuric acid as observed by optical microscopy under ambient conditions: (First row, images 1-8) Sequence of snapshots with increasing liquid volume induced by cooling the sample. The time interval $\Delta t$ between image 3 and image 4 is $\Delta t=40 \mathrm{~ms}$; the remaining time intervals are of the order of several seconds. (Second row, images 9-16) Sequence of snapshots with decreasing liquid volume induced by heating the sample. The five snapshots from image 12 to image 16 are taken $\Delta t=40 \mathrm{~ms}$ apart and, thus, cover $160 \mathrm{~ms}$. (b) Droplet shapes as obtained by numerical minimization of the interfacial free energy: The ring (or annulus) has inner and outer radius $r_{<}$and $r>$, respectively, the ring width $L=r>-r<$, and the aspect ratio $a=r / r>=2 / 3$. The volume $V$ is varied from $V=0.5 L^{3}$ on the left to $V=22 L^{3}$ on the right. The droplet height $h$ is measured in units of $L$ and color coded as indicated by the horizontal bar on the left. The contact angles are $\theta_{,}=0^{\circ}$ and $\theta_{\delta}=105^{\circ}$ for all values of $\mathrm{V} / \mathrm{L}^{3}$ apart from the shape for $V / L^{3}=5.95$. In the latter case, the contact angle $\theta_{o}$ was taken to be $\theta_{o}=150^{\circ}$ in order to show a bulge with a pinned inner contact line.

In order to find nonaxisymmetric droplet morphologies, the interfacial free energy was minimized by numerical algorithms implemented in the software "SURFACE EVOLVER" version 2.14. ${ }^{29}$ This numerical procedure starts from a triangulation of the $(\alpha \beta)$ interface. The interfacial energy of the model surface then depends on $3 N$ coordinates where $N$ is the number of vertices of the triangular mesh. Global and local constraints imposed on the configuration reduce the number of independent degrees of freedom. A constant volume of the model droplet, for instance, is guaranteed by a global constraint, whereas additional local constraints keep the vertices of the contact line in the plane of the substrate. A conjugate gradient descent method was used to find local minima of the interfacial free energy.

\section{Results and Discussion}

Sequence of Morphologies. An example for the experimentally observed time evolution of the droplet morphology is shown in Figure 1a. This figure consists of a series of video images of the droplet morphology on top of a single annular $(\gamma)$ domain. In the upper series of images, the liquid volume is increased by cooling the substrate; in the lower series of images, this volume is decreased by heating. The images represent snapshots at subsequent times $t$. To visualize the process, subsequent snapshots correspond to time intervals $\Delta t$ of several seconds if the droplets grow or shrink without changing their shape whereas shorter time intervals $\Delta t$ of $40 \mathrm{~ms}$ were chosen close to morphological transitions.

Direct inspection of Figure la shows that the first three images 1-3 represent ringlike axisymmetric channels. Between image 3 and image 4, a morphological wetting transition takes place: the axisymmetry is broken and the liquid channel develops a single bulge. This bulge spreads onto the lyophobic $(\delta)$ domain enclosed

(29) Brakke, K. The surface evolver. Exp. Muth. 2000, 87, 7768-7775. by the lyophilic $(\gamma)$ annulus. For a given annulus, this transition can be repeated many times, and the bulge typically develops each time at the same position. This broken symmetry presumably arises from the presence of small substrate defects or impurities which act as nucleation sites for the bulge formation.

In our experiments, the bulge detaches from the inner $(\gamma \delta)$ domain boundary. By further cooling, the bulge still grows inward as shown by images $5-7$ in Figure la until it covers the whole interior $(\delta)$ domain. It then forms a spherical cap as shown by image 8. Further cooling leads to an increase of the droplet volume and the height of the spherical cap while the contact line stays pinned to the outer $(\gamma \delta)$ boundary until the contact angle $\theta$ reaches the limiting value $\theta=\theta_{\delta}$ of the hydrophobic $(\delta)$ domains. A further volume increase then leads to a spreading of the droplet onto the exterior $(\delta)$ domain. Thus, during cooling, we observe ringlike axisymmetric channels $(\mathrm{R})$, channels with a single bulge (B), and spherical caps (C).

As the sample is heated up again, the droplet volume decreases as shown in the lower series of images 9-16 in Figure la. First, one observes Newton's rings as in images 9 and 10 which move to the center and indicate that the droplet becomes flatter. After a while, the droplets appear only dark, and the contact line detaches from the outer $(\gamma \delta)$ domain boundary and moves inward as shown in images 11 and 12 .

Finally, after the contact line has transversed the interior $(\delta)$ domain, the liquid redistributes and forms again an axisymmetric channel which sits on top of the lyophilic $(\gamma)$ annulus. Images 12-16 in Figure la are again separated by time intervals $\Delta t=40 \mathrm{~ms}$ and include the transient morphologies 13-15. For the example displayed in Figure la, the transition from the spherical cap (C) to the ringlike axisymmetric channel (R) occurs via intermediate bulge states (B). In some cases, we did not observe such intermediate bulge states, but all intermediate states were nonaxisymmetric. 

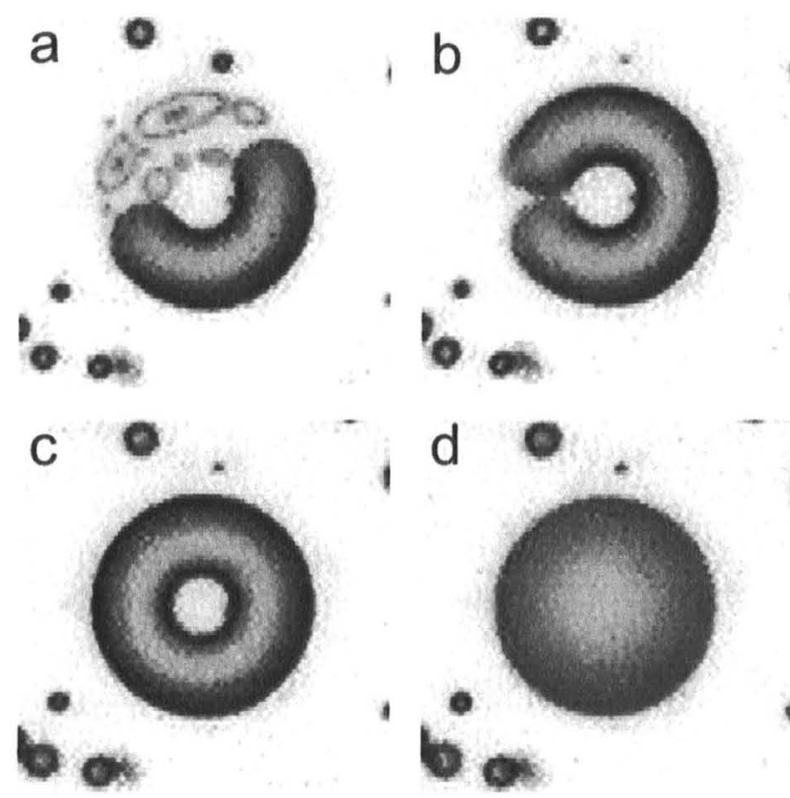

Figure 2. Sequence of optical micrographs for wetting morphologies of aqueous sulfuric acid, the volume of which is increased from (a) to (d) by cooling the sample, for an annular surface domain with width $L=(5.40 \pm 0.08) \mu \mathrm{m}$ and aspect ratio $a=0.294 \pm$ 0.007 . The two images (c) and (d) are $\Delta y=40 \mathrm{~ms}$ apart. No bulge morphology (B) is observed. At low volumes, the closed ringlike liquid channel breaks up and forms an open channel with two ends, as shown in (a) and (b).

Whenever a stable bulge appeared, it remained at a fixed position. Thus, we did not observe a thermally activated diffusion of the bulge as discussed in ref 22 . We believe that this is related to surface inhomogeneities or defects which act as pinning sites and suppress such a diffusive motion.

For broader rings, a different scenario was encountered. In Figure 2a, a typical sequence of morphologies observed on a lyophilic $(\gamma)$ annulus with a width of $L=(5.40 \pm 0.08) \mu \mathrm{m}$ and aspect ratio $a=0.294 \pm 0.007$ is displayed for the same type of cooling experiment as described above. In images (a) and (b), the amount of condensed liquid is not sufficient to cover the whole $(\gamma)$ annulus and one observes an open ringlike channel (Ro). In Figure 2c, a closed ringlike channel $(R)$ has been formed which then transforms into a spherical cap as shown in image (d). The time interval between image (c) and (d) is $\Delta t=40 \mathrm{~ms}$. We never observed the bulge state (B) for these broad ring geometries.

AFM Measurements. In order to determine the volume and the morphology of the condensed liquid and to compare the wetting morphologies with numerical calculations, those liquid morphologies that were stable for hours were studied by AFM measurements at room temperature.

The volume of the condensed liquid was determined in two ways. First, the AFM gray scale image of the liquid droplet was integrated on the whole ring area by an image processing program, the background was subtracted, and the difference was multiplied by a calibrated pixel volume. Second, the volume was estimated by fitting the images to simple geometrical shapes such as spherical caps and cylinder segments. The volume measurements obtained by these two methods agreed typically to within $5 \%$

Some examples for the wetting morphologies determined by the AFM measurements are shown in Figure 3. A typical example for a ringlike channel is shown in Figure $3 \mathrm{a}$ and $\mathrm{b}$ which represent a perspective view and gray scale height image of this morphology. In this case, the height $h$ of the channel is $h \simeq 150 \mathrm{~nm}$ and its width is $L=(1.25 \pm 0.08) \mu \mathrm{m}$. This corresponds to a volume of $V \simeq 2.6 \mu \mathrm{m}^{3}$ as obtained by image processing, and of $V \simeq 2.7 \mu \mathrm{m}^{3}$ as estimated from numerical shape calculations. In Figure $3 c$, an AFM image of a bulge state is shown. The ringlike region of this latter state has the height $h \simeq 230 \mathrm{~nm}$ while the height of the bulge is $h \simeq 830 \mathrm{~nm}$ which leads to a volume of $V=(7.6 \pm 0.3) \mu \mathrm{m}^{3}$ by image processing, where $65 \%$ of the volume is contained in the bulge. The cap-shaped droplet in Figure $3 \mathrm{~d}$ has a volume of $V \simeq$ $12.7 \mu \mathrm{m}^{3}$ and $V \simeq 13.8 \mu \mathrm{m}^{3}$ as obtained by image processing and numerical shape calculations, respectively. The cross sections through this latter shape can be well fitted by circular segments; see the data at the bottom of Figure 3d. Thus, the shape of this droplet is well described by a spherical cap.

Shape Calculations. Minimizing the interfacial free energy by analytical and numerical methods, we find ringlike channels (R), spherical caps $(\mathrm{C})$, and channels with a pronounced bulge (B) as stable droplet morphologies on the lyophilic annulus; see Figure Ib. Open ringlike channels (Ro), as experimentally observed by light microscopy (see heating sequence in Figure 1, second row, as well as Figure $2 \mathrm{a}$ and $\mathrm{b}$ ), are obtained from our numerical minimizations for lyophilic contact angles $\theta_{\gamma}>0^{\circ}$.

For the special case $\theta_{\gamma}=0^{\circ}$, the $(\gamma)$ domain is always completely covered by the liquid as predicted in ref 30 for all liquid morphologies which are curved "outward", that is, toward the vapor phase, corresponding to a positive pressure differenc $P_{\beta}-P_{\alpha}$ We find spherical caps $(\mathrm{C})$ as well as closed ringlike channels (R) which are both axisymmetric with respect to the center of the lyophilic annulus. Note that the $(\alpha \beta)$ interface of a ringlike channel $(\mathrm{R})$ is a doubly connected surface in contrast to the simply connected $(\alpha \beta)$ interface of the spherical cap (C) and of the open channel (Ro).

The analytical calculations revealed that two different types of closed axisymmetric channels $(R)$ exist on a lyophilic ring with $\theta_{\gamma}=0^{\circ}$ which correspond to extrema of the interfacial free energy. For both types of channel states, the outer contact line is always attached to the outer $(\gamma \delta)$ domain boundary. However, the inner contact line is either attached to the inner $(\gamma \delta)$ domain boundary or is located entirely within the interior $(\delta)$ domain. The configuration with the free contact line on the interior $(\delta)$ domain turns out to be mechanically unstable. Our results are consistent with those obtained in ref 17 for the "clamped doughnut geometry" in the limit of small contact angles.

A nonaxisymmetric bulgelike configuration (B) has been found by numerical minimization of the interfacial free energy for fixed droplet volume; see Figure $\mathrm{lb}$. The liquid is not evenly distributed around the center of the lyophilic ring but forms a single bulge. The $(\alpha \beta)$ interface of the bulgelike shape (B) and the ringlike channel (R) have the same topological genus, which cannot be used to distinguish between these two configurations. Such a distinction is provided, for example, by the position of the center of mass of the liquid. Another order parameter, which is sensitive to a transition between these morphologies, is the moment of inertia of the liquid with respect to the symmetry axis of the annular $(\gamma)$ domain.

We find two different types of stable bulgelike morphologies (B) depending on the volume of the droplet and the lyophobicity of the $(\delta)$ domains. For small volumes, the inner contact line of the bulge remains attached to the $(\gamma \delta)$ domain boundary, while for large volumes it is located within the interior $(\delta)$ domain. In

(30) Valencia, A.; Brinkmann, M.; Lipowsky, R. Liquid bridges in chemically structured slit pores. Langmuir 2001, 17, 3390-3399 

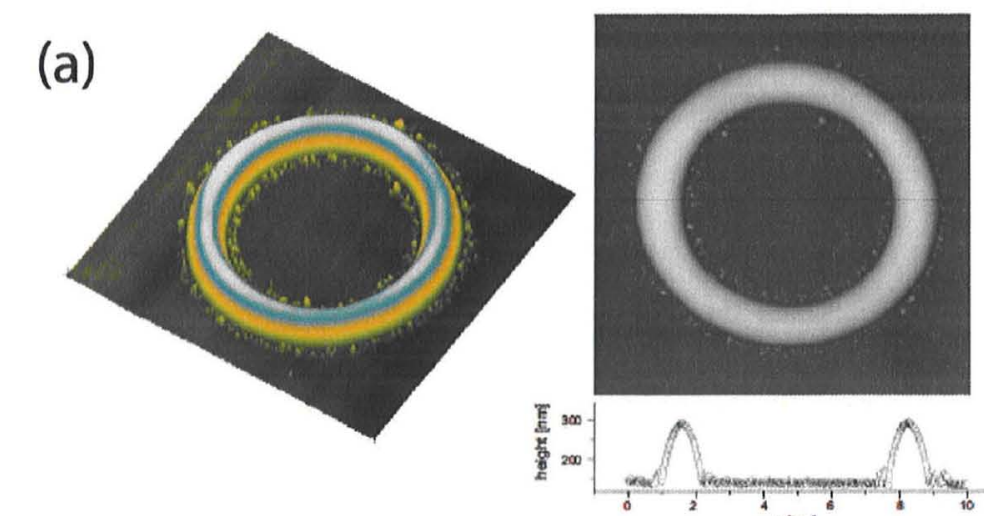

(b)
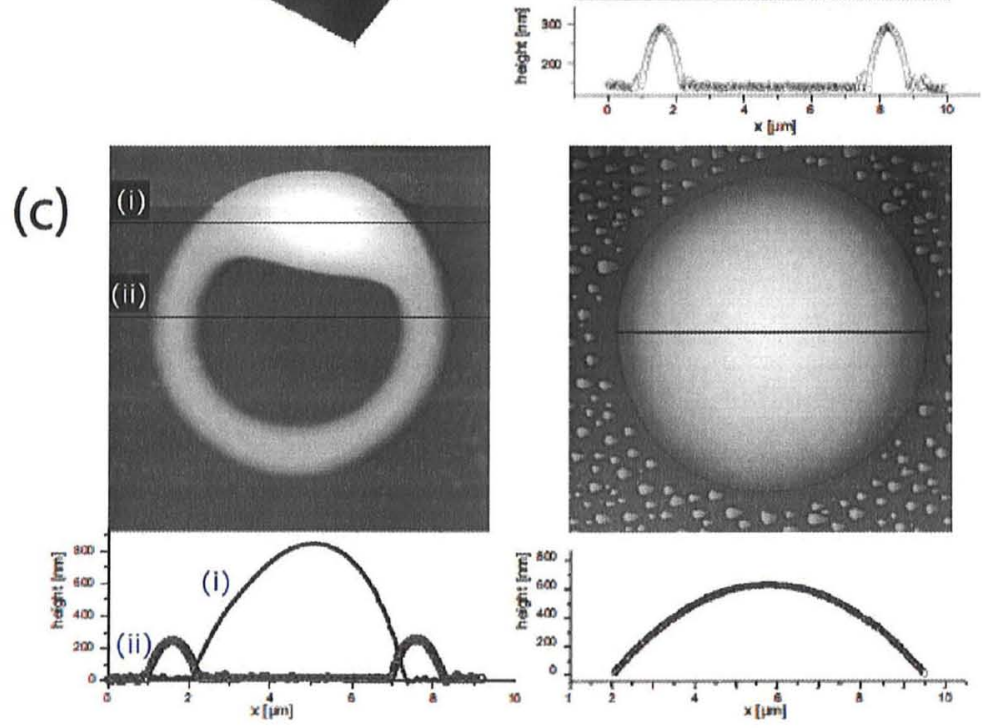

Figure 3. Wetting morphologies as observed by AFM in tapping mode: (a) Oblique and (b) top view of a ringlike channel morphology. (c) Top view of a liquid channel with a bulge; the two cross sections (i) and (ii) are taken perpendicular to the substrate surface. (d) Top view of a spherical cap with one such cross section.

our experiments on broad rings with an aspect ratio of $a=0.294 \pm$ 0.007 and estimated contact angles $\theta_{\gamma}=33^{\circ}$ and $\theta_{\delta}=105^{\circ}$, we found no locally stable configuration of the channel morphology (B) with a bulge.

Morphological Diagram. The morphology of a liquid droplet on a given lyophilic ring depends (i) on the wettabilities, that is, the equilibrium contact angles of the liquid on the $(\gamma)$ and $(\delta)$ domains, respectively, (ii) on the aspect ratio of the inner and outer radius of the $(\gamma \delta)$ domain boundary, and (iii) on the droplet volume. One convenient way to summarize the different shapes or types of droplets is by drawing a morphological diagram depending on the control parameters of the system. As basic control parameters of our system, we have considered the aspect ratio $a=$ $r<r>$ of the radii and the rescaled volume $v=V / L^{3}$. The contact angle on the lyophobic domains was chosen to be the experimentally measured value $\theta_{\delta}=105^{\circ}$, while the contact angle on the lyophilic domains was taken to be $\theta_{\gamma^{\prime}}=0^{\circ}$. Using both analytical and numerical calculations, we have determined regions of parameter values $(a, v)$ for which certain droplet morphologies appear as locally or globally stable configurations with respect to the interfacial free energy.

For aspect ratios $a \lesssim 0.34$ and rescaled droplet volumes $v \lesssim$ 1.70, only axisymmetric droplets occur as shown in Figure 4. At higher values of the control parameters $a$ and $v$, a "triple point" $\left(a^{\mathrm{tn}}, v^{\mathrm{tp}}\right) \simeq(0.44,2.45)$ exists. At this point, a ringlike liquid channel $(\mathrm{R})$, a channel with a bulge (B), and a spherical cap (C) have the same interfacial free energy. The full lines represent transition lines, that is, the set of points $(a, v)$ in the space of control parameters for which two droplet morphologies have the same interfacial free energy. In the present case, the morphological diagram exhibits three transition lines which meet in the "triple point" $\left(a^{\mathrm{tp}}, v^{\mathrm{tp}}\right)$.

The two experimentally investigated geometries correspond to the vertical lines in Figure 4. These geometries represent narrow rings with an aspect ratio of $a=0.667 \pm 0.016$ and broad rings with $a=0.294 \pm 0.007$. In agreement with the experiments, all three droplet morphologies are present on the narrow rings, whereas no stable bulge morphology exists on the broader rings. In the latter case, the transition from the ringlike configuration to the spherical cap takes place directly without an intermediate bulge morphology.

Stability. The dashed lines shown in the morphological diagram of Figure 4 represent instability lines (in close analogy to spinodal lines in bulk phase diagrams). Thus, if one starts with a certain liquid morphology and moves the system across a transition line in the morphogical diagram, this morphology remains locally stable until one reaches the corresponding instability line. At the latter line, the local minimum of the interfacial free energy disappears and the unstable morphology must decay into another stable one. Starting, for example, from the ringlike configuration ( $R$ ) at $a \gtrsim 0.44$ and increasing the volume, we cross the shape transition line as shown as a full line in Figure 4. Beyond this transition line, the symmetric ringlike droplet shape $(R)$ does not represent the global minimum of the interfacial free energy but is still mechanically metastable. Reaching the dashed instability line, the ringlike channel morphology ( $R$ ) will decay into the (B) channels with a bulge. 


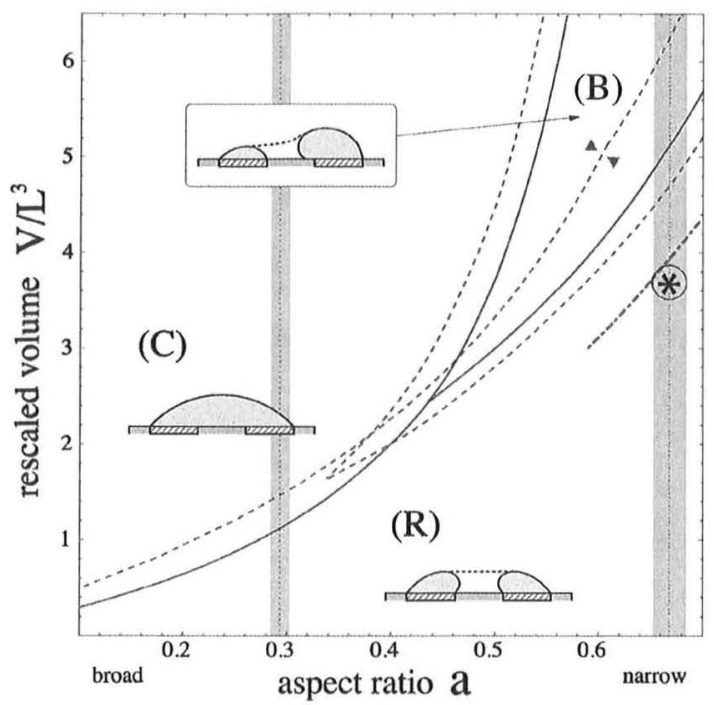

Figure 4. Morphological diagram for a liquid droplet of volume $V$ in contact with a lyophilic ring (or annulus), which has inner radius $r_{<}$, outer radius $r$, width $L=r>-r_{<}$, and aspect ratio $a=$ $r</ r_{>}$. The contact angles on the lyophilic $\gamma$ ring and the lyophobic $\delta$ substrate are $\theta_{\gamma}=0^{\circ}$ and $\theta_{\delta}=105^{\circ}$, respectively. Three different droplet morphologies can be distinguished: a ringlike channel (R) with both inner and outer contact line attached to the domain boundaries of the lyophilic ring; a nonaxisymmetric chamnel with a bulge (B), the inner contact line of which may be partially located on the lyophobic disk; and a spherical cap (C) spanning both the lyophilic ring and the lyophobic disk. Full and dashed lines correspond to transition and instability lines, respectively, as explained in the text. The shaded vertical stripes represent the experimentally investigated geometries of narrow rings with $a=0.667 \pm$ 0.16 and broad rings with $a=0.294 \pm 0.007$. The two adjacent triangles indicate a small kink of the corresonding instability line. The encircled asterisk corresponds to the parameter values for the smallest (B) channels with a bulge as observed by AFM. For the reduced contact angle $\theta_{\delta}=90^{\circ}$, the (B) channels become unstable along the dashed-dotted line.

In general, we expect that a ringlike channel $(\mathrm{R})$ with a detached, "free" inner contact line located on the inner $(\delta)$ domain will always be unstable. On broad rings this instability of the $(\alpha \beta)$ interface is related to an axisymmetric soft mode, while narrow rings are characterized by a nonaxisymmetric soft mode. The existence of two instability mechanisms reflects the fact that there are two global minima, (C) and (B), along the instability line of (R), which exhibit different symmetries. Our findings are consistent with earlier results for the "clamped doughnut" geometry obtained in ref 17 and for the hole formation in liquid films in ref 31 . However, the latter studies are restricted to small gradients in the height of the $(\alpha \beta)$ interface above the substrate, that is, to droplet shapes without overhangs, while our analysis is valid for arbitrary droplet shapes.

The two different instability mechanisms lead to a slight kink in the instability line of $(\mathrm{R})$ for $a \simeq 0.61$ as indicated in Figure 4 by the two adjacent triangles. For $a \gtrsim 0.61$, the $(\alpha \beta)$ interface becomes unstable with respect to a soft mode with both contact lines attached to the $(\gamma \delta)$ domain boundary. In this case, the contact angle $\theta_{\text {in }}$ along the inner contact line is smaller than $\theta_{\delta}$. For $a \lessgtr 0.61$, on the other hand, the contact angle $\theta_{\text {in }}$ will reach the value $\theta_{\delta}$ before the $(\alpha \beta)$ interface becomes unstable for pinned

(3I) Srolovitz, D. J.; Safran, S. A. Capillary instabilities in thin films: I Energetics. I. Appl. Phys. 1986, 60, 247-254. contact lines. As a consequence, the inner contact line starts to slide onto the domain $(\delta)$. Such an axisymmetric ringlike droplet $(R)$ is unstable and can only be seen as a transient configuration. It decays and forms either a spherical cap (C) or a (B) channel with a bulge.

In our calculations, we used $\theta_{\gamma}=0$ which implies that spherical caps $(C)$ are locally stable for arbitrarily small volumes since the liquid has to wet the lyophilic ring completely as proven in ref 30. As a consequence, no dashed lines appear below the transition lines for spherical caps $(\mathrm{C})$ in the morphological diagram of Figure 4. In our experiments with $\theta_{\gamma}>0$, on the other hand, the contact line of a sufficiently flat cap (C) detaches from the outer $(\gamma \delta)$ domain boundary, which can lead to an asymmetric configuration of the liquid phase $(\beta)$ as shown in image 12 of Figure la. In addition, for fairly high volumes, yet another transition takes place, at which the outer contact line of the caplike droplet $(\mathrm{C})$ moves onto the lyophobic domain $(\delta)$. This latter transition has not been included in the morphological diagram in Figure 4.

The measured volumes for the ringlike channel correspond to parameter values, for which the ring configuration is stable according to Figure 4. The smallest bulgelike droplets (B) as observed by AFM are indicated in Figure 4 by an encircled asterisk. Inspection of this figure shows that this encircled asterisk is located below the instability line of the (B) morphology for contact angle $\theta_{\delta}=105^{\circ}$ and close to this instability line for $\theta=90^{\circ}$. Thus, we can explain the observation of these small (B) droplets if we replace the equilibrium value of the contact angle $\theta$ by its receding value as appropriate for a decreasing droplet volume and assume that this receding angle is smaller than $90^{\circ}$. Observations of the dynamics of the transition from a ringlike channel to a bulge and vice versa reveal a large hysteresis between both morphologies.

For $\theta_{\gamma}=0$ as used in the present calculations, the ringlike channel $(\mathrm{R})$ has to wet the lyophilic annulus completely for arbitrarily small volumes. ${ }^{30}$ For $\theta_{\gamma}>0$, on the other hand, additional small-volume morphologies appear in close analogy to lyophilic stripes. ${ }^{13}$ First, for sufficiently small volume, the liquid forms a small spherical cap that is located within the lyophilic annulus. As one increases the liquid volume, the spherical droplet grows until its contact line reaches the domain boundaries of the annulus. A further volume increase then leads to elongated liquid channels with two end caps. The latter morphologies have been experimentally observed as shown in Figure 2a and b. Increasing the volume even further, the two end caps fuse and form the ringlike channel as shown in Figure 2c. Therefore, for $\theta_{\gamma}>0$, the morphology diagram contains additional transition and instability lines that truncate the ringlike channel morphology $(\mathrm{R})$ at small volumes but are absent from the diagram in Figure 4 for $\theta_{\gamma}=0$.

\section{Conclusions and Summary}

The morphology of liquid droplets (aqueous sulfuric acid) deposited onto lyophilic rings within lyophobic substrates has been investigated experimentally by optical microscopy as well as by AFM for two geometries, narrow rings with aspect ratio $a=$ $0.667 \pm 0.016$ and broader rings with aspect ratio $a=0.294 \pm$ 0.007. Upon variation of the volume of the adsorbed liquid, several volume-induced morphological transitions are observed. Experimentally, we find five different morphological phases: partially wetted rings, ringlike channels with uniform cross sections, ringlike channels with a bulge, spherical caps with a pinned contact line, and spherical caps with a depinned contact line. The different shapes agree well with analytically and numerically calculated morphologies for the same geometry. While the 
bulge phase is observed on narrow rings, it does not occur for broader rings, consistent with the numerically calculated morphological diagram as shown in Figure 4, which contains both the transition lines, at which two droplet morphologies have the same interfacial free energy, and the instability lines, at which a certain morphology attains its limit of metastability.

The morphological diagram contains a triple point at which all three morphologies have the same free energy. In the vicinity of

(32) Kusumaatmaja, H.; Léopoldès, J.; Dupuis, A.; Yeomans, J. M. Drop dynamics on chemically patterned surfaces. Europhys. Lett. 2006, 73, 740-746. (33) Valencia, A.; Lipowsky, R. Nucleation through a double barrier for : chenically patterned substrate. Langmuir 2004, 20, 1986-1996.

(34) Blecua, P.; Lipowsky, R.; Kierfeld, J. Line tension effects for liquid droplets on circular surface domains. Lamgmmir 2006, 22, 11041-11059.

(35) Winter, D.: Virnau, P.: Binder, K. Monte Carlo test of the classical theory for heterogeneous nucleation barriers. Phys. Rev. Let1. 2009, 103, 225703. this point, one expects to see interesting nucleation and spreading processes related to the different metastable morphologies. These dynamical phenomena could be studied, for example, using lattice Boltzmann methods as applied to contact angle hysteresis in ref 32. Other interesting issues include the nucleation of droplets on ring-shaped surface domains, which can be governed by two free energy barriers as for circular domains, ${ }^{33}$ as well as line tension effects ${ }^{34}$ that will become important for sufficiently small surface domains in the nanoregime. The latter effects should also be accessible to Monte Carlo methods as recently used to study nucleation at uniform substrate surfaces. ${ }^{3.5}$

Acknowledgment. Support by the Deutsche Forschungsgemeinschaft via the Schwerpunktprogramm on wetting and the Stb 513 is gratefully acknowledged. 